\title{
An Application of Graph Theory to the Electrical Circuit Using Matrix Method
}

\author{
Samai'la Abdullahi \\ Department of mathematics, sokoto state university, sokoto P.M.B 2134, Sokoto, Nigeria.
}

\begin{abstract}
A graph is a pair of two set $V$ and $E$ so that $G=(V, E)$. A graph is a pictorial representation of a system using two basic element nodes and edges, a node is represented by a circle (either hallo shade) and edge is represented by a line segment connecting two nodes together. In this paper we present a circuit network in the concept of graph theory application and also circuit models of graph are represented in logical connection method were we formulate matrix method of adjacency and incidence of matrix and application of truth table.
\end{abstract}

Key word: Euler Circuit and Path, Graph representation of Circuit networks, Representation of Graph models, Representation Of circuit network using logical truth table.

\section{Introduction}

The world graph is refers to a specific mathematics structure usually represented as a diagram consist of point joint by line. In this paper, we present the graph as a worthy way of of presenting circuit networks of electrical circuit. In section 2, we recall some definitions from circuit so that the representation of circuit network will make sense. In section 3, how to represent circuit network as a graph. In section 4, is the central one the describes how to apply graph theory to model the circuit network. In section 5, we illustrate the circuit networks by working out the test example.

\section{Definitions}

CIRCUIT METWORK: A circuit is a path which ends at the vertex it begins. An electric circuit is a closed loop formed by source, wires, load, and a switch, when switch is turned on the electrical circuit is complete and current flows from negative terminals of the power source. An electrical circuit is categories in to three type namely series, parallel and series and parallel circuit.

EULER CIRCUIT: Euler circuit in a graph $\mathrm{V}(\mathrm{V}, \mathrm{E})$ is a closed path containing every edge of $\mathrm{G}(\mathrm{V}, \mathrm{E})$.

EULER PATH: Euler path in a graph $\mathrm{G}(\mathrm{V}, \mathrm{E})$ is a path containing every edge of $\mathrm{G}(\mathrm{V}, \mathrm{G})$.

\section{Graph Representation Of Circuit Networks}

The representation of graph in circuit network are one of the type of representation of graph in which the current flows in circuit and present the linking of connection between resistors series and parallel connection are determined in the circuit.

The representations are

\section{Figure 1}

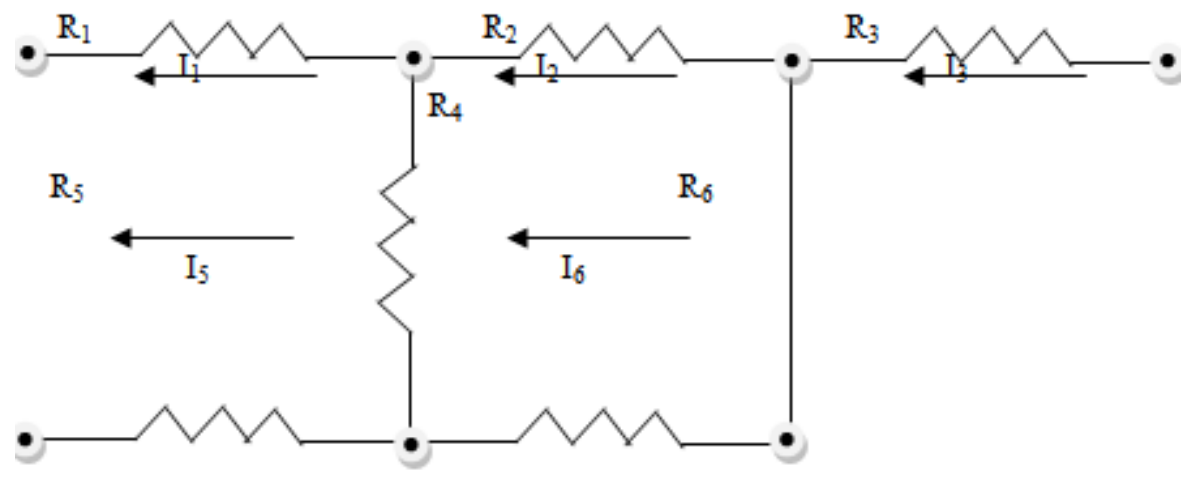


The schematic diagram of the electric circuit network

\section{FIGURE 2}
$\mathrm{R}_{1}$
$\mathrm{R}_{2}$
$\mathrm{R}_{3}$

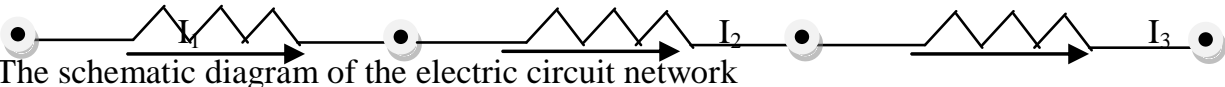

\section{Representation Of Graph Model}

A graph model is used to represented circuit network in graph model by tracing the verties of the circuit and edges contain in the given circuit.

The graph models are

\section{Figure}

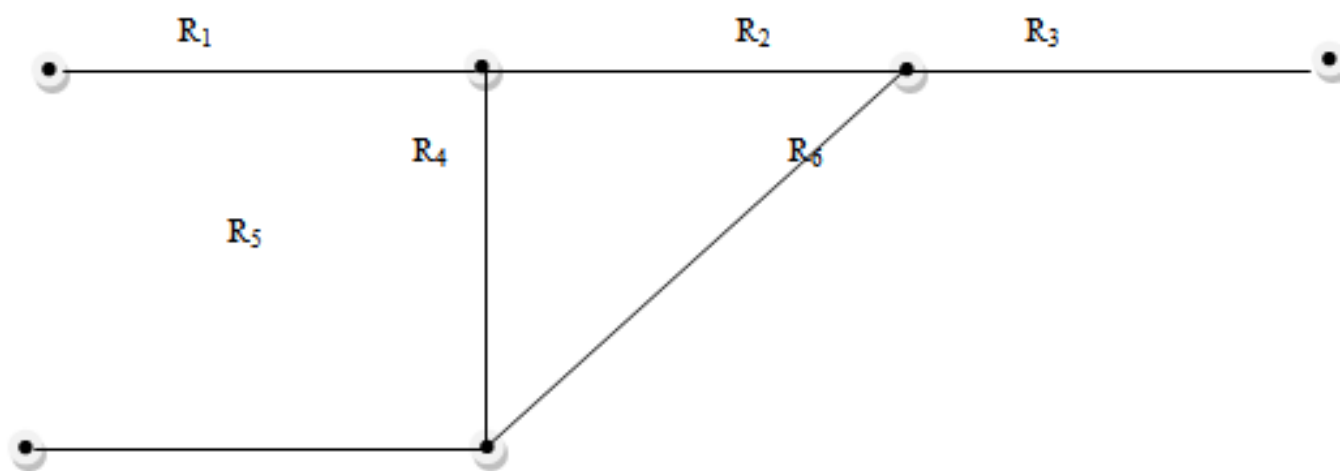

An undirected graph model representation of the circuit network with its edges, weight corresponding to the resistor value of each branch

Figure 2
A $\quad \mathrm{R}_{1}$
B $\quad \mathrm{R}_{2}$
C $\quad \mathrm{R}_{3}$

An undirected graph model representation of the circuit network with its edges, weight corresponding to the resistor value of each branch.

\section{Graph representation of matrices}

A graph can actually be represented using matrices method the two of the most widely used matrices for graph representation are adjacency and incidence matrices. An adjacency matrix is a square matrix in which each row and column is represented by a vertex.

Consider figure 1 as an example it has six vertices $V=\left\{R_{1}, R_{2}, R_{3}, R_{4}, R_{5}, R_{6}\right\}$ this mean that the square matrix must be $6 \times 6$ let each row and column is represented by each of the six vertices in $\mathrm{V}$.

\begin{tabular}{|l|r|r|r|r|r|r|}
\hline & LOGICAL TRUTH TABLE \\
\hline R1 & & R2 & R3 & R4 & R5 & R6 \\
\hline R2 & 0 & 1 & 0 & 0 & 0 & 0 \\
\hline R3 & 1 & 0 & 1 & 0 & 0 & 1 \\
\hline R4 & 0 & 1 & 0 & 1 & 0 & 1 \\
\hline R5 & 0 & 0 & 1 & 0 & 0 & 0 \\
\hline R6 & 0 & 0 & 0 & 0 & 0 & 1 \\
\hline
\end{tabular}

the adjacency matrix generated by $6 \times 6$ square matrix and represent by

$$
\mathrm{G}=
$$

$$
\begin{array}{llllll}
0 & 1 & 0 & 0 & 0 & 0 \\
1 & 0 & 1 & 0 & 0 & 1 \\
0 & 1 & 0 & 1 & 0 & 1 \\
0 & 0 & 1 & 0 & 0 & 0 \\
0 & 0 & 0 & 0 & 0 & 1 \\
0 & 1 & 1 & 0 & 1 & 0
\end{array}
$$


Also in figure 21 as an example it has three vertices $V=\left\{R_{1}, R_{2}, R_{3}\right\}$ this mean that the square matrix must be $3 \times 3$ let each row and column is represented by each of the six vertices in $\mathrm{V}$.

LOGICAL TRUTH TABLE
\begin{tabular}{|l|r|r|r|}
\hline & \multicolumn{1}{|l|}{ R1 } & R2 & \multicolumn{1}{|c|}{ R3 } \\
\hline R1 & 0 & 1 & 0 \\
\hline R2 & 1 & 0 & 1 \\
\hline R3 & 0 & 1 & 0 \\
\hline R4 & 0 & 0 & 1 \\
\hline R5 & 0 & 0 & 0 \\
\hline R6 & 0 & 1 & 1 \\
\hline
\end{tabular}

The adjacency matrix generated by $3 \times 3$ square matrix and represent by

$$
\mathrm{G}=\left|\begin{array}{lll}
0 & 1 & 0 \\
1 & 0 & 1 \\
0 & 1 & 0
\end{array}\right|
$$

\section{Conclusion}

So far, the research attention has been focuses on the graph of electrical circuit network, the graph theory has a wide application in various field of science and engineering course especially in electrical and computer engineering and also in communication industry.

A circuit network can be represented by different metho which include

- Circuit representation

- Graph model representation

- $\quad$ Matrix method by using logical truth table

\section{Reference}

[1] B. kosko, Neural Network and Fuzzy System: A Dynamical Systems Approch to machine Intelligence, Englewood Cliffs, NJ: Prentice - Hall, 1992

[2] Y. Yao and W.J. Freeman, "Model of biological pattern recognition with spatially chaotic dynamics, " Neural Networks, vol 2. No. 2 ,1990.pp. 153-170

[3] J.J. Hopfied "Neural Networks and physical systems with emergent collective computational abilities," proceeding of the National Academy ofscience, vol. 79,1982,pp.2554-2558. 